\title{
Efecto del aporte térmico en la resistencia a la corrosión de un recubrimiento de acero inoxidable 414-N depositado por soldadura FCAW
}

\section{Welding heat input effect on the corrosion resistance of a 414-N stainless steel cladding deposited by FCAW welding}

\author{
H.G. Carreón Garcidueñas, A. Medina Flores \\ Instituto de Investigación en Metalurgia y Materiales, Universidad Michoacana de San Nicolás \\ Morelia, 58030 Michoacán, México. \\ E. Huape Padilla*, L. Béjar Gómez \\ Facultad de Ingenieria Mecánica, Universidad Michoacana de San Nicolás de Hidalgo \\ Morelia, 58030 Michoacán, México.
}

(Received: January 16th, 2019; Accepted: December 5th, 2019)

\begin{abstract}
En el proceso de colada continua, los rodillos se utilizan para la contención, soporte, conducción y transporte de la losa. Para extender substancialmente la vida útil de los rodillos, la superficie está cubierta con una capa resistente al desgaste llamada revestimiento duro el cual es aplicado por medio de soldadura de recargue, para mejorar las superficies que se encuentran sometidas a desgaste severo, oxidación o corrosión de los rodillos. El objetivo del presente trabajo es estudiar el efecto del aporte térmico por medio de técnicas electroquímicas de un acero inoxidable martensítico 414N aplicado como revestimiento duro en agua de mar sintética. El proceso de soldadura para la aplicación de los recargues fue por medio de soldadura de arco con electrodo tubular (FCAW) variando el voltaje del arco. Las técnicas electroquímicas fueron polarización potenciodinámica (PP) y espectroscopia de impedancia electroquímica (EIS). Los resultados indican que todos los recargues presentan una estructura martensítica de listón, donde $26 \mathrm{~V}$ presentó un $5.44 \%$ de ferrita, $28 \mathrm{~V} 5.33 \%$ y $30 \mathrm{~V}$ $5.0 \%$, se puede observar que con un alto aporte térmico empleado se obtiene la menor cantidad de ferrita. De los resultados electroquímicos se puede observar, para $30 \mathrm{~V}$ se tiene el comportamiento más activo teniendo un $\mathrm{E}_{\text {corr }}$ de $-535 \mathrm{mV} / \mathrm{ECS}$, siendo para $26 \mathrm{~V}$ y $28 \mathrm{~V}$ los valores más nobles (-380 mV/ECS y $-425 \mathrm{mV} / \mathrm{ECS})$ respectivamente. Para los 28 y $30 \mathrm{~V}$ se presentan potenciales de ruptura de la pasividad (313 $\mathrm{mV} / \mathrm{ECS}$ y $132 \mathrm{mV} / \mathrm{ECS})$ respectivamente.
\end{abstract}

In continuous casting process, rollers are used for the containment, support, conduction and transport of the slab. To substantially extend the life of the rollers, the surface is covered with a wear-resistant coating called hardfacing which is applied by means of recharge welding to improve the surfaces that are subject to severe wear, oxidation or corrosion of the surfaces. The objective of this work is to study the effect of welding heat input on the corrosion resistance of a martensitic stainless steel AISI 414-N applied as a hardfacing in synthetic seawater. Welding process for the application of the recharges was FCAW. The electrochemical techniques were potentiodynamic polarization (PP) and electrochemical impedance spectroscopy (EIS). The results indicate that all the hardfacing have a lath martensitic structure, where $26 \mathrm{~V}$

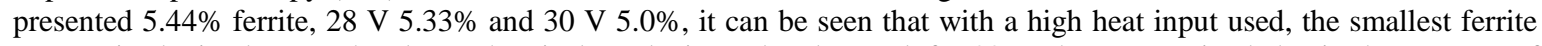
amount is obtained. From the electrochemical results it can be observed, for $30 \mathrm{~V}$, the most active behavior has an $\mathrm{E}_{\text {corr }}$ of $-535 \mathrm{mV} / \mathrm{ECS}$, being for $26 \mathrm{~V}$ and $28 \mathrm{~V}$ the noblest values $(-380 \mathrm{mV} / \mathrm{ECS}$ and $-425 \mathrm{mV} / \mathrm{ECS})$ respectively. For 28 and $30 \mathrm{~V}$ passivity rupture potentials $(313 \mathrm{mV} / \mathrm{ECS}$ and $132 \mathrm{mV} / \mathrm{ECS})$ are presented, respectively.

\section{Introduction}

En el proceso de colada continua, los rodillos se utilizan para la contención, soporte, conducción y transporte de la losa. Una instalación típica contiene entre 350 y 700 rodillos los cuales se encuentran sujetos a diferentes tipos de desgaste dependiendo de su posición durante la colada; es decir, en la parte superior de la línea, predomina la corrosión húmeda debido a sistemas de enfriamiento de los rodillos [1]. Para extender substancialmente la vida útil de los rodillos, la superficie está cubierta con una capa resistente al desgaste llamada "revestimiento duro" el cual es aplicado por medio de soldadura de recargue. Los revestimientos duros han sido utilizados ampliamente para mejorar las superficies que se encuentran sometidas a desgaste severo, oxidación o corrosión de los rodillos. Son varios los materiales aplicados como revestimientos duros, siendo los aceros inoxidables martensíticos los más utilizados [2,3]. Los aceros inoxidables martensíticos presentan excelentes propiedades mecánicas pero una moderada resistencia a la corrosión, la cual es afectada cuando se encuentra en un medio acuoso [4].

En este sentido, el estudio de los consumibles y procesos de soldadura son de interés para la optimización y desarrollo de los revestimientos duros; por ejemplo, los revestimientos duros aplicados por soldadura de fricción han ido teniendo gran aceptación. Sin embargo, no se ha entendido por completo los mecanismos de las superficies de fricción [3,5]. La soldadura por arco eléctrico es el proceso más utilizado para aplicar los revestimientos duros. La tendencia es hacia procesos semi-automáticos como la soldadura con electrodo tubular (FCAW) o procesos automáticos como la soldadura con arco metálico y protección gaseosa (GMAW), así como al proceso manual de soldadura con electrodo revestido (SMAW) especialmente para aplicaciones en campo. 
El aporte térmico $(\mathrm{HI})$ es una de las variables importantes que se refiere a la calidad del cordón de soldadura, este depende de la Tensión (V), Intensidad (I), Velocidad de soldadura (v) y la eficiencia de transferencia de calor en el proceso ( $\eta$ ). Debido al calor aportado y al enfriamiento que se presenta durante la soldadura, la formación de tensiones residuales que se generan son perjudiciales para las propiedades mecánicas y/o de corrosión en servicio, por lo que es conveniente realizar algún tratamiento térmico o mecánico (precalentamiento, relevado de esfuerzos, etc.) [6]. Durante las décadas de los 80's y 90's, se introdujo como un estándar en las acereras los revestimientos duros aplicados con electrodos tubulares de acero inoxidable martensítico AISI 420, mejorando la vida útil de los rodillos de colada. El acero AISI 414 es un derivado del AISI 420, se caracteriza por presentar un rendimiento superior, pero al paso del tiempo exhibe un deterioro inaceptable. Una variante del AISI 414 se presenta con la adición de Nitrógeno como sustituto del carbono, inhibiendo el crecimiento de grano mejoran la resistencia a la corrosión por la formación de nitruros [7].

El objetivo del presente trabajo es estudiar con técnicas electroquímicas el efecto del aporte térmico en el acero inoxidable martensítico $414 \mathrm{~N}$ aplicado como recubrimiento con la técnica de soldadura de arco con electrodo tubular, la evaluación se realiza en presencia de agua de mar sintética

\section{Experimental}

\section{Procedimiento de soldadura}

El proceso de soldadura para la aplicación de los recargues fue por medio de soldadura de arco con electrodo tubular (FCAW) con una fuente de poder WAP 650-00, con características de CC/CV con promedio de $650 \mathrm{~A}, 44 \mathrm{~V} \mathrm{DC}$ al $100 \%$ en ciclo de servicio. Para la manipulación de las variables secundarias como longitud del stick-out, velocidad de alimentación del alambre, desplazamientos longitudinal y transversal, velocidad de oscilación, se utilizó una consola digital con función modular. Los principales parámetros utilizados en el proceso de soldadura se muestran en la Tabla 1.

Los recubrimientos fueron aplicados en posición plana con la siguiente secuencia: 1 capa de recubrimiento de $430 \mathrm{~N}$ y dos capas posteriores de $414 \mathrm{~N}$ donde cada capa consta de 3 cordones de recargues sobre un metal base AISI 4140, como se muestra en la Figura 1. Posteriormente, se cortaron las placas en un sentido longitudinal para obtener muestras de $10 \times 10 \times 10 \mathrm{~mm}^{3}$ aproximadamente para llevar a cabo las pruebas de corrosión en una solución de agua de mar sintética. La composición química de los elementos

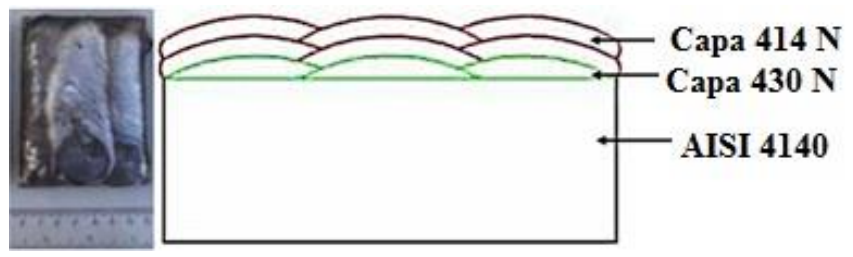

Figura 1. Secuencia de la soldadura de recargue.
Tabla 1. Parámetros de soldadura.

\begin{tabular}{ccccc}
\hline Muestra & $\begin{array}{c}\text { Voltaje } \\
(\mathrm{V})\end{array}$ & $\begin{array}{c}\text { Corriente } \\
(\mathrm{A})\end{array}$ & $\begin{array}{c}\text { Velocidad de } \\
\text { soldadura } \\
(\mathrm{mm} / \mathrm{s})\end{array}$ & $\begin{array}{c}\text { Stick-out } \\
(\mathrm{mm})\end{array}$ \\
\hline S1 & 26 & 300 & 2.0 & 28 \\
S2 & 28 & 300 & 2.0 & 28 \\
S3 & 30 & 300 & 2.0 & 28 \\
\hline
\end{tabular}

Tabla 2. Análisis químicos de los electrodos tubulares.

\begin{tabular}{cccccccc}
\hline Electrodo & $\mathbf{C}$ & $\mathbf{N}$ & $\mathbf{M n}$ & $\mathbf{S i}$ & $\mathbf{C r}$ & $\mathbf{N i}$ & Mo \\
\hline $414 \mathrm{~N}$ & 0.12 & 0.12 & 1.51 & 0.58 & 12.86 & 3.09 & 0.45 \\
$430 \mathrm{~N}$ & 0.13 & 0.07 & 1.38 & 0.52 & 13.21 & 2.58 & 0.42 \\
\hline
\end{tabular}

presentes en los metales de aporte se muestra en la Tabla 2.

\section{Aporte térmico}

Durante el proceso de soldadura, no todo el calor generado por el arco puede ser utilizado por el proceso de fusión, los valores de eficiencia varían entre 20 y $85 \%$ en la soldadura por arco, siendo la conducción, la convección, la radiación y las salpicaduras, los responsables de las pérdidas.

El aporte térmico se calculó mediante la siguiente ecuación:

$$
H I=\eta \frac{I V}{v}
$$

Donde:

$H I=$ aporte térmico $(\mathrm{J} / \mathrm{mm})$.

$I=$ corriente de soldadura (A).

$V=$ voltaje de arco (V).

$v=$ velocidad de soldadura $(\mathrm{mm} / \mathrm{s})$.

$\eta=$ eficiencia de transferencia de calor en el proceso (0.8 para FCAW) [8].

La eficiencia de transferencia de calor $\eta$, es definida como sigue:

$$
\eta=\frac{Q}{Q_{\text {nominal }}}
$$

donde Q es la velocidad de transferencia de calor de la fuente de calor a la pieza de trabajo, $Q_{\text {nominal }}$ es la potencia nominal de la fuente de calor. Una parte de la energía proporcionada por la fuente de calor se transfiere a la pieza de trabajo y la parte restante se pierde en el entorno. Consecuentemente $\eta<1$. Los valores de la eficiencia de transferencia de calor para diferentes procesos se dan en la Tabla 3.

Tabla 3. Eficiencias térmicas para diferentes procesos de soldadura.

\begin{tabular}{cc}
\hline Proceso & Eficiencia, $\boldsymbol{\eta}$ \\
\hline SAW & 1 \\
SMAW & 0.8 \\
FCAW & 0.8 \\
MIG/MAG & 0.8 \\
TIG & 0.6 \\
\hline
\end{tabular}


Con los aportes térmicos utilizados para cada muestra, se determinaron los porcentajes de ferrita en las microestructuras, estos porcentajes fueron obtenidos por medio de la ecuación 2, la cual indica la regresión que relaciona el porcentaje de ferrita con el voltaje y velocidad de soldadura [9]:

$$
\mathrm{F}(\%)=5.63-0.16 \mathrm{~V}+0.34 v-0.12 \mathrm{~V}^{2}+0.05 \mathrm{~V} v+0.13 v^{2}
$$

Donde:

$$
\begin{aligned}
& \mathrm{F}=\text { ferrita, } \% \\
& \mathrm{~V}=\text { voltaje de arco, } \mathrm{V} \\
& v=\text { velocidad de soldadura, } \mathrm{mm} / \mathrm{s}
\end{aligned}
$$

\section{Inspección por Líquidos penetrantes}

Fue realizada una inspección por el método de líquidos penetrantes de acuerdo a los criterios de aceptación y/o rechazo especificados en el código aplicado (ANSI/AWS D1.1. STRUCTURAL 2004). Las indicaciones reportadas corresponden a las encontradas después de 20 minutos de haber sido aplicado el revelador a las probetas representativas para cada ensayo. La inspección fue realizada antes y después de haber sido rectificada la superficie de los recubrimientos.

\section{Técnicas electroquímicas}

Las pruebas electroquímicas fueron realizadas sobre el último cordón del recubrimiento de acero inoxidable martensítico $414 \mathrm{~N}$, para simular las condiciones del agua de mar, el electrolito con $\mathrm{pH} 8.2$ se preparó con agua destilada y reactivos grado analítico. Las pruebas electroquímicas fueron realizadas usando un potenciostato/galvanostato/ZRZ de ACM controlado por computadora. Todos los potenciales fueron medidos usando un electrodo de Calomel Saturado (SCE) como electrodo de referencia y una barra de grafito como electrodo auxiliar, las mediciones se llevaron a $19 \pm 0.5^{\circ} \mathrm{C}$ y el volumen de la solución fue de $60 \mathrm{ml}$. Las técnicas electroquímicas fueron polarización potenciodinámica y espectroscopia de impedancia electroquímica (EIS). Las curvas de polarización potenciodinámica fueron obtenidas variando el potencial aplicado con respecto al potencial de circuito abierto (OCP) de $-1500 \mathrm{mV}$ hasta $+1500 \mathrm{mV}$ a una velocidad de polarización de $1 \mathrm{mV} / \mathrm{s}$ de acuerdo con la norma ASTM G314. Un tiempo de retardo de $60 \mathrm{~min}$ fue programado hasta que se consiguió una lectura estable, antes de que el estudio electroquímico comenzara. Las pruebas de impedancia se llevaron a cabo usando una señal con una amplitud de $20 \mathrm{mV}$ vs el OCP entre un intervalo de frecuencia de $0.05 \mathrm{~Hz}$ a $10 \mathrm{kHz}$ obteniendo una medida a los $30 \mathrm{~min}, 12$ y $24 \mathrm{~h}$ de inmersión.

\section{Resultados y discusión}

En las Figuras 2a) y 2b), se puede observar la inspección por líquidos penetrantes de las placas soldadas para la condición de recargue y maquinadas, respectivamente. En la condición de recargue las muestras S1 y S2 tuvieron indicaciones mínimas de porosidades, las cuales fueron eliminadas durante el maquinado para la obtención de las

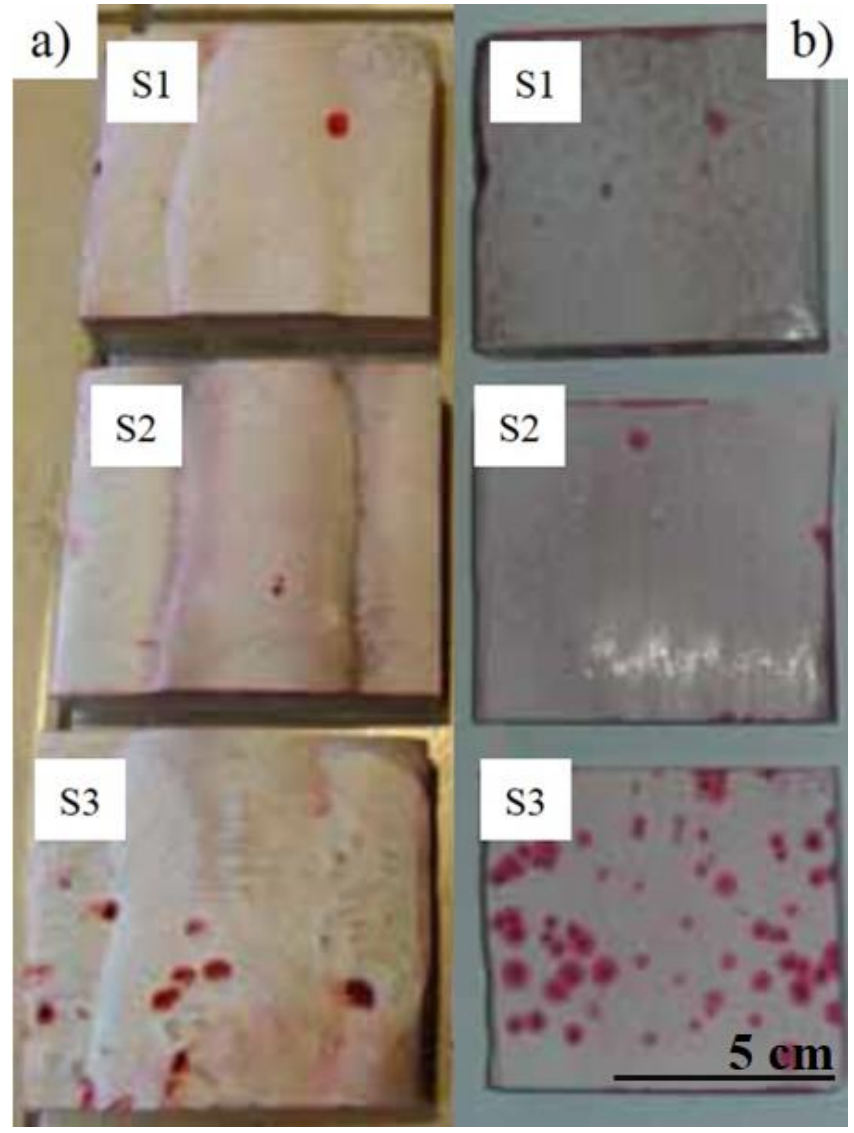

Figura 2. Inspección visual por líquidos penetrantes de los recubrimientos depositados a diferentes voltajes.

muestras para corrosión. Por otro lado, la muestra S3 presentó porosidades relevantes tanto en condición de recargue como después del maquinado, estas indicaciones externas relevantes superficiales son consideradas inaceptables conforme a los criterios de aceptación y/o rechazo del código aplicado (ANSI/AWS D1.1 STRUCTURAL 2004). Mientras que las muestras S1 y S2 presentan indicaciones no relevantes.

\section{Caracterización estructural}

De acuerdo con el diagrama de fases para un acero inoxidable martensítico con $13 \%$ de $\mathrm{Cr}$, a una temperatura de $1050^{\circ} \mathrm{C}$ la austenita y los carburos se encuentran estables; conforme se va enfriando a temperatura ambiente la austenita se transforma a martensita permaneciendo los carburos en la microestructura. Estos carburos son del tipo M7C3 ricos en Cr, la precipitación de estos carburos en los límites de grano se puede atribuir a la migración de los átomos de $\mathrm{Cr}$ vecinos a los límites de grano, agotando los átomos de $\mathrm{Cr}$ de la matriz y exponiendo a la corrosión intergranular si se agota el $\mathrm{Cr}$ por debajo del valor crítico necesario para la pasivación. Los resultados obtenidos, indican que todos los recargues presentan una estructura martensítica de listón, donde para $26 \mathrm{~V}$ presentó un $5.44 \%$ de ferrita, para $28 \mathrm{~V}$ un $5.33 \%$ y para $30 \mathrm{~V}$ un $5.0 \%$, se puede observar que con un alto aporte térmico empleado se obtiene la menor cantidad de ferrita. La microestructura presente a temperatura ambiente está compuesta de ferrita y carburos 


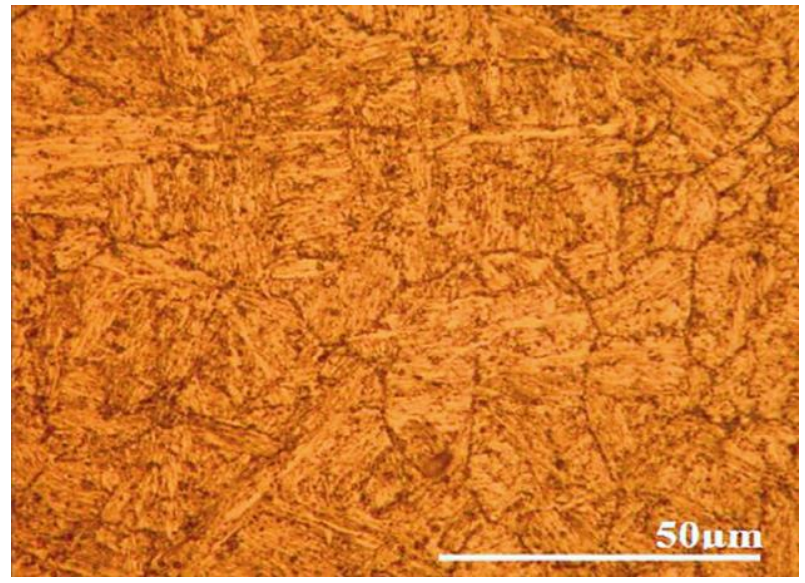

Figura 3. Micrografía del acero inoxidable 414-N en condición de recargue.

en los límites de grano en una matriz de martensita (ver Figura 3), coincidiendo por la reportada por Abbasi [10].

\section{Técnicas electroquímicas}

En las curvas de polarización potenciodinámicas, Figura 4, se puede observar para $\mathrm{S} 1$ un comportamiento activo de disolución a partir de potencial de corrosión -380 mV/ECS, posteriormente tiene una región de pseudo-pasivación en un rango entre -282 a $144 \mathrm{mV} / \mathrm{ECS} ; \quad \mathrm{S} 2$ presenta un comportamiento activo de disolución en el rango de -425 a $233 \mathrm{mV} / \mathrm{ECS}$ partiendo del potencial de corrosión, continuando con un comportamiento de pasivación de -233 hasta $-59 \mathrm{mV} / \mathrm{ECS}$ y pseudo-pasivación de -59 hasta $323 \mathrm{mV} / \mathrm{ECS}$, también se puede apreciar un potencial de picadura a $313 \mathrm{mV} / \mathrm{ECS}$. Para S3 se puede ver un comportamiento de disolución partiendo del potencial de corrosión -534 hasta $-261 \mathrm{mV} / \mathrm{ECS}$ seguido de una zona de pasivación entre -261 y 95mV/ECS, en un potencial de $140 \mathrm{mV} / \mathrm{ECS}$. En este sentido, Saadi [11] reporta un potencial de descomposición o potencial de picadura característico para los aceros inoxidables sometidos a una solución de $\mathrm{NaCl}$ por debajo de la región transpasiva en la cual la densidad de corriente aumenta por encima de la densidad de corriente pasiva.

Los valores de potencial de corrosión $\left(E_{\text {corr }}\right)$ y densidad de

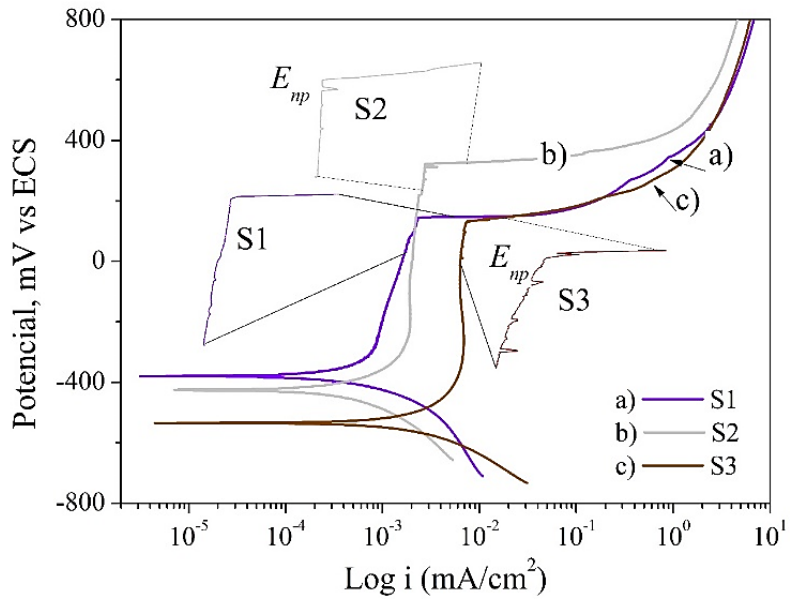

Figura 4. Curvas potenciodinámicas para a) $\mathrm{S} 1(26 \mathrm{~V})$, b) $\mathrm{S} 2(28 \mathrm{~V})$ y c) $\mathrm{S} 3$ $(30 \mathrm{~V})$.

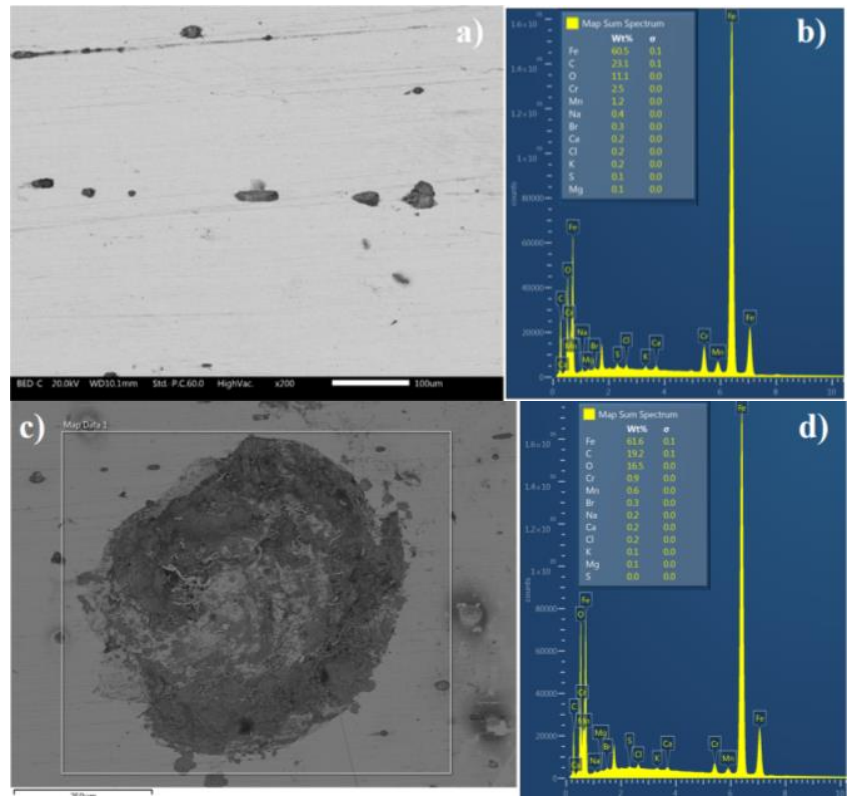

Figura 5. Imágenes MEB de la superficie corroída de S3: a) picaduras con productos formados, b) EDS productos internos en la picadura, c) producto de corrosión formado en la superficie y d) EDS del producto de corrosión formado en la superficie.

corriente $\left(i_{\text {corr }}\right)$ obtenidos por el método de intercepción de TAFEL se muestran en la Tabla 4. Las pendientes de Tafel fueron determinadas por medio de un arreglo lineal de los datos de polarización en un rango de $\pm 50 \mathrm{mV}$ del $E_{\text {corr }}$.

\section{Caracterización de superficies corroídas}

En la Figura 5a) se pueden observar imágenes MEB de la superficie corroída de la muestra S3, donde se aprecian las picaduras formadas durante las pruebas electroquímicas, lo cual coincide con el comportamiento de la Figura 4c, dentro de las picaduras se formaron productos de corrosión de acuerdo con los análisis EDS (Figura 5b) se detectaron O, ( $\mathrm{Fe}, \mathrm{Cr}$ y $\mathrm{Mn}$ ) elementos del electrodo, así como $\mathrm{C}, \mathrm{Cl}, \mathrm{Ca}$, $\mathrm{Na}$ y $\mathrm{Mg}$ elementos presentes en la solución de agua de mar sintética. La Figura 5c, muestra la imagen de MEB del producto de corrosión formado en la superficie de la muestra,

Tabla 4. Parámetros electroquímicos obtenidos de las curvas de polarización.

\begin{tabular}{cccc}
\hline Muestra & S1 & S2 & S3 \\
\hline $\begin{array}{c}E_{\text {corr }} \\
(\mathrm{mV} / \mathrm{ECS}) \\
i_{\text {corr }} \\
\left(\mu \mathrm{A} / \mathrm{cm}^{2}\right)\end{array}$ & -380 & -425 & -534 \\
$\begin{array}{c}\text { Enp } \\
(\mathrm{mV} / \mathrm{ECS})\end{array}$ & 0.288 & 0.375 & 1.54 \\
$\begin{array}{c}\beta \mathrm{a} \\
(\mathrm{mV} / \text { década })\end{array}$ & 151 & 313 & 140 \\
$\begin{array}{c}\text { Bc } \\
(\mathrm{mV} / \text { década }) \\
\text { Ferrita } \\
(\%)\end{array}$ & 80 & 139 & 183 \\
$\begin{array}{c}\text { Aporte térmico } \\
(\mathrm{kJ} / \mathrm{mm})\end{array}$ & 3.12 & 3.36 & 140 \\
\hline
\end{tabular}



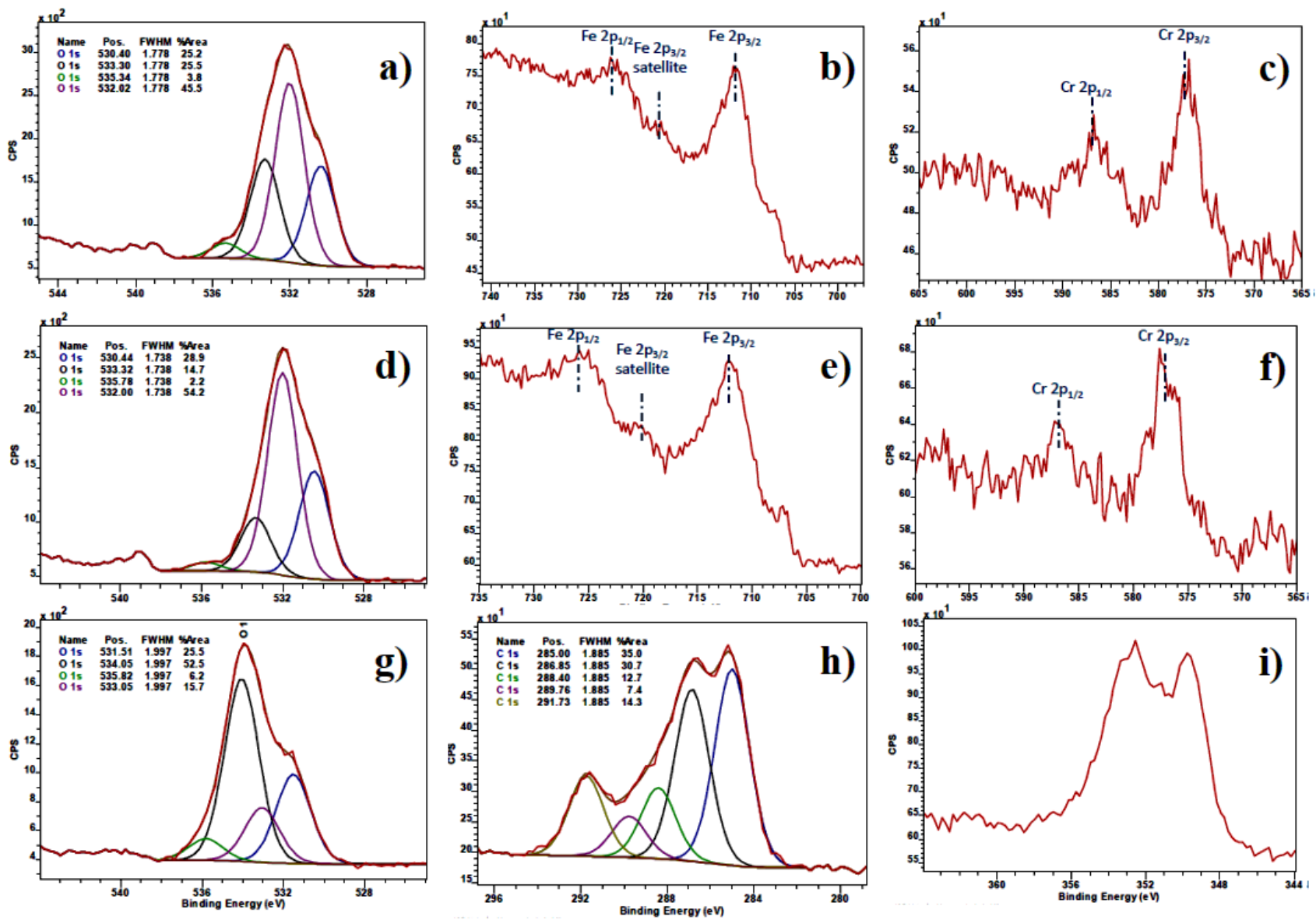

Figura 6. Curvas de espectroscopia fotoelectrónica de rayos X para: S1 a) O, b) Fe y c) Cr; S2 d) O, e) Fe y f) Cr; S3 g) O, h) C y i) Ca.

en el cual se aprecia un incremento en la presencia del $\mathrm{O}$ (Figura 5d).

Las superficies corroídas en agua de mar sintética fueron analizadas por medio de la técnica de espectroscopia fotoelectrónica de rayos X (XPS) para caracterizar los productos de corrosión obtenidos durante los ensayos. Para las muestras S1 y S2, se observan que las formas y composición de los espectros de $\mathrm{O}$ en el nivel 1s son muy similares para ambas muestras Figuras 6a) y 6d), respectivamente, la presencia de óxido, (oxi) hidróxido y agua se puede deducir a partir del ajuste máximo de $\mathrm{O} 1 \mathrm{~s}$. Los picos a $\sim 540 \mathrm{eV}$ se originan a partir del oxígeno molecular $\left(\mathrm{O}_{2}\right)$ debido al aire ambiente. Las formas de los espectros para el Fe (Figuras $6 \mathrm{~b}$ y $6 \mathrm{e}$, respectivamente) en el nivel $2 p$ son muy comparables para ambas posiciones, la presencia de óxido, (oxi) hidróxido y hierro metálico se puede deducir de $\mathrm{Fe} 2 \mathrm{p}_{3 / 2}$. De igual manera, la forma de los espectros del $\mathrm{Cr}$ (Figuras 6c y 6f, respectivamente) en el nivel $2 p$ muy comparables para ambas posiciones para la presencia de óxido, hidróxido y $\mathrm{Cr}^{0}$ metálico se puede deducir de $\mathrm{Cr} 2 \mathrm{p}_{3 / 2}$. En cuanto al hierro, se necesitaría un ajuste de pico más sofisticado para identificarlos y separarlos de forma confiable $[12,13]$. La naturaleza de las diferentes especies de $\mathrm{O} 1 \mathrm{~s}$ para la muestra $\mathrm{S} 3$ (Figuras $6 \mathrm{~g}$, 6h y $6 \mathrm{i}$ ), se podría contribuir a varios óxidos $(\mathrm{Ca}, \mathrm{Mg}, \mathrm{Si}, \ldots)$, la forma y composición del espectro de $\mathrm{C}$ en el nivel $1 \mathrm{~s}$ es completamente diferentes de las muestras S1 y S2, una nueva contribución adicional a $292 \mathrm{eV}$ puede ser de carbonatos $\mathrm{MgCO}_{3} / \mathrm{CaCO}_{3}$. Para el $\mathrm{Ca}$ en el nivel $2 \mathrm{p}$ dominado por un doblete en $\sim 352 \mathrm{eV}$. La forma de pico no encaja perfectamente con un $2 p$ que indica algunas contribuciones adicionales. Probablemente de Magnesio (línea de Auger $\mathrm{L}_{1} \mathrm{~L}_{23} \mathrm{a} \sim 350 \mathrm{eV}$ ).

Durante la disolución anódica en condiciones neutras y alcalinas en un ambiente marino, está bien establecido que se presenta un proceso donde se consume el oxígeno disuelto generando los iones hidroxilo, de acuerdo con la siguiente reacción:

$$
\mathrm{O}_{2}+2 \mathrm{H}_{2} \mathrm{O}+4 e^{-} \rightarrow 4 \mathrm{OH}^{-}
$$

Con la producción de iones hidroxilo se presenta un aumento en el pH del electrolito cercano a la superficie del recubrimiento. De este modo, el aumento del $\mathrm{pH}$ en el agua de mar resultará en la precipitación de sales insolubles; tales como, $\mathrm{CaCO}_{3}, \mathrm{Mg}(\mathrm{OH})_{2}$, y estos procesos se pueden describir mediante las siguientes reacciones [14,15]:

$$
\begin{aligned}
& \mathrm{OH}^{-}+\mathrm{HCO}_{3}^{-} \rightarrow \mathrm{CO}_{3}^{-}+\mathrm{H}_{2} \mathrm{O} \\
& \mathrm{Ca}^{2+}+\mathrm{CO}_{3}^{2-} \rightarrow \mathrm{CaCO}_{3} \\
& \mathrm{Mg}^{2+}+2 \mathrm{OH}^{-} \rightarrow \mathrm{Mg}(\mathrm{OH})_{2}
\end{aligned}
$$

Estas reacciones dan lugar a la formación de un depósito calcáreo sobre la superficie del acero. Donde la formación preferencial de carbonato de calcio en lugar de carbonato de 

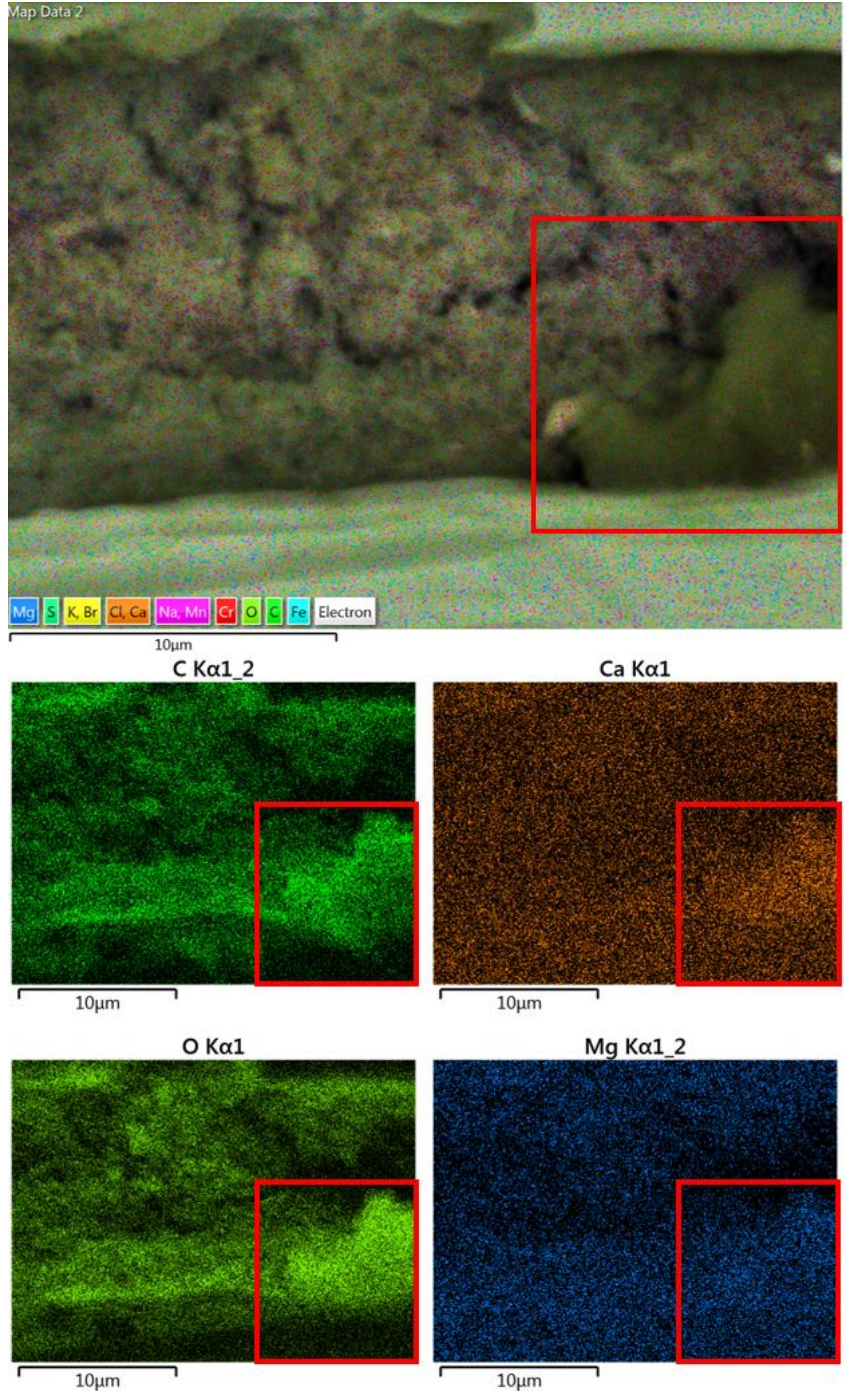

Figura 7. Mapeos por EDS de los productos de corrosión depositados en las picaduras para los elementos $\mathrm{C}, \mathrm{Ca}, \mathrm{O}$ y $\mathrm{Mg}$.

magnesio puede explicarse por la solubilidad mucho mayor del carbonato de magnesio, esto se puede apreciar en la Figura $3 \mathrm{~h}$. Elbeik et al., encontraron que para un acero suave una película de $\mathrm{CaCO}_{3}$ es depositada cuando se tiene un potencial de corrosión de $-620 \mathrm{mV} / \mathrm{ECS}$ en agua de mar sintética [15], este comportamiento es muy similar para S3 donde se obtuvo el valor más próximo de $-534 \mathrm{mV} /$ ECS del potencial de corrosión y de acuerdo a la Figura 5c) fue la única muestra que presento la formación de productos de

Tabla 5. Resultados obtenidos por el método EIS para las muestras S1 y S2 en una solución de agua de mar sintética.

\begin{tabular}{|c|c|c|c|c|c|c|}
\hline \multirow{2}{*}{ Muestra } & \multirow{2}{*}{$\begin{array}{c}R_{S} \\
\left(\Omega \mathrm{cm}^{2}\right)\end{array}$} & \multirow{2}{*}{ 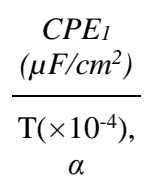 } & \multirow{2}{*}{$\begin{array}{c}R_{p} \\
\left(\Omega \mathrm{cm}^{2}\right)\end{array}$} & \multirow{2}{*}{ 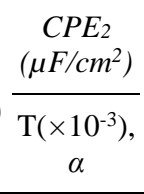 } & \multirow{2}{*}{$\begin{array}{c}W_{R} \\
\left(\Omega \mathrm{cm}^{2}\right)\end{array}$} & \multirow{2}{*}{$\begin{array}{c}R_{t c} \\
\left(\Omega \mathrm{cm}^{2}\right)\end{array}$} \\
\hline & & & & & & \\
\hline S1 & 31 & $\begin{array}{c}1.024 \\
0.82\end{array}$ & 33288 & $\begin{array}{c}4.451 \\
0.98\end{array}$ & ----- & 27410 \\
\hline S3 & 28.76 & $\begin{array}{c}8.505 \\
0.5\end{array}$ & 810 & $\begin{array}{l}12.4 \\
0.39\end{array}$ & 19118 & 20.22 \\
\hline
\end{tabular}
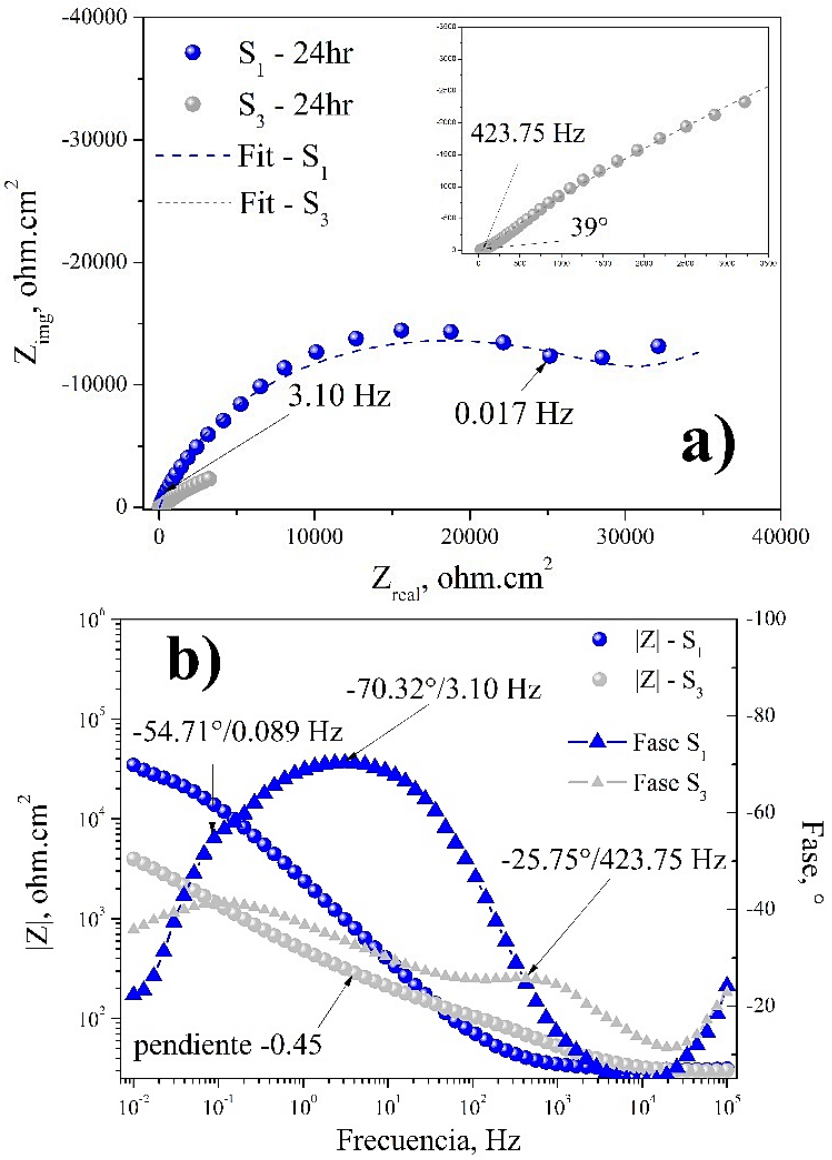

Figura 8. Diagramas a) Nyquist y b) Bode, para las muestras S1 y S3.

corrosión. En este sentido, también encontraron que una película de $\mathrm{CaCO}_{3}$ uniformemente distribuida, presenta una buena efectividad en los aceros suaves actuando como una película protectora, debido a que reduce en gran medida la difusión del oxígeno a la superficie del acero. La presencia de $\mathrm{CaCO}_{3}$ en la superficie de la muestra $\mathrm{S} 3$, puede apreciarse en el comportamiento de pasivación (entre -261 y $95 \mathrm{mV} /$ ECS) que presentó en la curva de polarización de la Figura 4c. En la Figura 7 se pueden observar los mapeos de EDS para el $\mathrm{C}, \mathrm{Ca}, \mathrm{O}$ y $\mathrm{Mg}$, en ellos se aprecia claramente una zona con mayor concentración de $\mathrm{Ca}, \mathrm{C}$, y $\mathrm{O}$, de acuerdo con los resultados de XPS se trata de carbonatos $\mathrm{CaCO}_{3} \mathrm{O}$ $\mathrm{MgCO}_{3}$.

La Figura 8 muestra los resultados EIS de las soldaduras S1 y S3. Los circuitos equivalentes que se utilizan para modelar los resultados de EIS se muestran en la Fig. 9 y los parámetros extraídos según el modelo se presentan en la Tabla 5. El espectro de S1 (Figura 8a) presenta un comportamiento capacitivo en frecuencias altas teniendo una constante de tiempo, conforme la frecuencia disminuye muestra claramente la presencia de una segunda constante de tiempo; la primer constante de tiempo puede apreciarse claramente en la formación de un pico en frecuencias intermedias del ángulo de fase $\left(-70.32^{\circ} / 3.10 \mathrm{~Hz}\right)$ en la Figura $8 \mathrm{~b}$ ), lo que significa un comportamiento capacitivo debido a la formación de un óxido compacto protector, mientras que en frecuencias bajas se presenta un ensanchamiento del ángulo de fase $\left(-54.71^{\circ} / 0.089 \mathrm{~Hz}\right)$ 


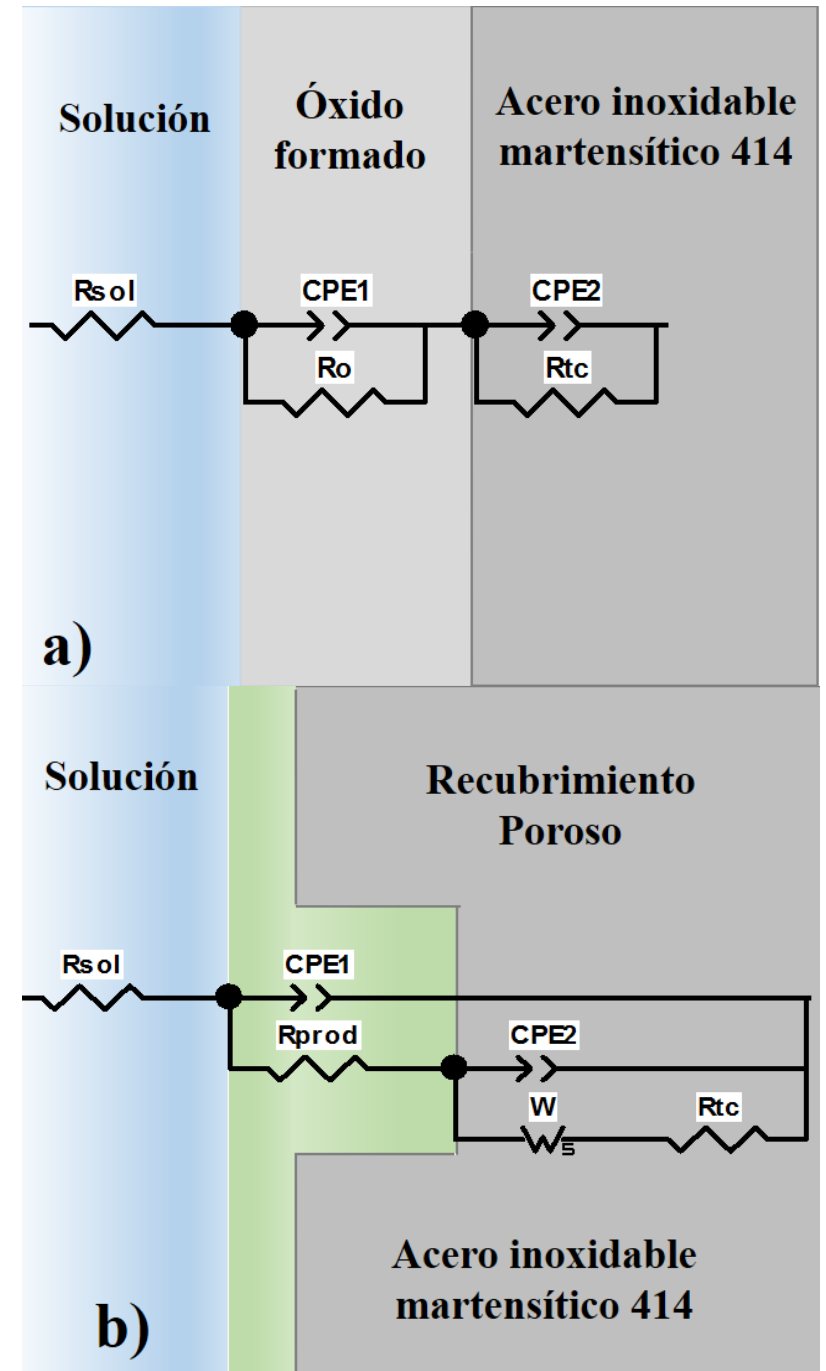

Figura 9. Circuitos equivalentes para el ajuste de los datos experimentales de las muestras: a) S1 y b) S3.

ocasionado por la formación de la segunda constante de tiempo; respecto a S1 en la Figura 9a) muestra el circuito equivalente generado por medio del software Zview con los parámetros extraídos, $\mathrm{CPE}_{1}$ explica la contribución dieléctrica de la película de óxido de cromo, mientras que Ro corresponde a esta resistencia de la película pasiva. Por otro lado, Imaz et al., propuso un circuito equivalente para un recubrimiento de $\mathrm{Cr}$ corroído en una solución acuosa de $\mathrm{NaCl}$ donde $\mathrm{CPE}_{2}$ representa la capacitancia de doble capa y Rtc representa la resistencia de transferencia de carga del proceso de corrosión a través de la superficie del recubrimiento [16].

Por otro lado, el espectro Nyquist de la muestra S3 (Figura 8a) presenta un comportamiento capacitivo desde frecuencias altas hasta $423.75 \mathrm{~Hz}$, aproximadamente; a partir de ésta frecuencia, el valor de la impedancia tiende a formar una línea recta con una inclinación de $39^{\circ}$ a bajas frecuencias. Éste comportamiento es debido a una respuesta difusional de tipo Warburg con tendencia semi-infinita, el cual puede comprobarse con el valor de la pendiente $(-0.45)$ en el diagrama de Bode [17]; ésta impedancia difusional está relacionada con la microestructura del recubrimiento, es decir los poros formados durante el recargue, la microestructura generada y los límites de grano pueden funcionar como canales de difusión para las especies reactivas [18,19], así como por la formación de una capa de difusión entre la solución y la superficie del recubrimiento. Respecto a S3 en la Figura 9b) muestra el circuito equivalente generado por medio del software Zview con los parámetros extraídos, donde $\mathrm{CPE}_{1}$ explica la contribución dieléctrica del producto de corrosión formado en la superficie mientras que $\mathrm{R}_{\text {pro }}$ corresponde a la resistencia del producto de corrosión. Por otro lado, $\mathrm{CPE}_{2}$ representa la capacitancia de debido a la presencia del poro, $R_{\mathrm{tc}}$ representa la resistencia de transferencia de carga en el poro y Ws es la impedancia de Warburg que describe el proceso de difusión semi-infinito.

Las impedancias de las constantes de tiempo CPE pueden expresarse por:

$$
Z=Y_{0}^{-1}(j \omega)^{-\alpha}
$$

Donde $Y_{0}$ es el módulo, $\omega$ es la frecuencia angular y $\alpha$ es la fase que indica lo siguiente: para $\alpha=1$ el CPE se comporta como un capacitor ideal con poca porosidad, para $\alpha=0$ el $\mathrm{CPE}$ se comporta como una resistencia, para $\alpha=0.5 \mathrm{CPE}$ es una impedancia de Warburg, para $\alpha=-1$, CPE es un inductor.

\section{Conclusiones}

Los resultados mostrados en este trabajo, indican que la variación en el voltaje del arco durante el proceso de soldadura, tiene un efecto significativo en la resistencia a la corrosión de los recubrimientos de aceros inoxidables martensíticos en agua de mar sintética sin un tratamiento térmico postsoldadura. Con el mayor aporte térmico $(30 \mathrm{~V})$, de acuerdo con la inspección por líquidos penetrantes el recubrimiento depositado presenta poros generados durante el proceso de soldadura, de igual manera la disolución del recubrimiento, así como la formación de picaduras durante el proceso de corrosión fue mayor. Un factor que es determinante para que presente la menor resistencia a la corrosión, es el porcentaje de ferrita el cual es el menor de los tres voltajes, permitiendo la formación de carburos ricos en $\mathrm{Cr}$ en los límites de grano como se mostró en la micrografía durante el enfriamiento lento al aire, los cuales pueden funcionar como canales de difusión de especies. Por otro lado, con el menor aporte térmico $(26 \mathrm{~V})$ se depositó un recubrimiento libre de poros en la superficie de acuerdo con la inspección visual, de igual manera no se presentó la formación de picaduras durante la prueba de corrosión. El recubrimiento tuvo el comportamiento más noble y el porcentaje de ferrita fue el mayor, la presencia de un óxido de cromo protector se formó de acuerdo con los resultados de XPS.

\section{Agradecimientos}

Los autores agradecen al Dr. Cuauhtémoc Maldonado y al M.C. Víctor M. Bedolla por su apoyo para la realización del presente trabajo. Las pruebas electroquímicas fueron realizadas en el Laboratorio de Degradación de Materiales - FIM Edificio J, Ciudad Universitaria. 


\section{Referencias}

[1]. R. Paschold, Svetsaren 1, 17 (2001).

[2]. A. Gualco, H. G. Svoboda, E. S. Surian, L. A. de Vedia, Mater. Des. 31, 4165 (2010).

[3]. R. Puli, G.D. Janaki Ram, Surf. Coat. Tech. 209, 1 (2012).

[4]. A. Toro, A. Sinatora, D.K. Tanaka, A.P. Tschiptschin, Wear 251, 1257 (2001).

[5]. R. Puli, G.D. Janaki Ram, Surf. Coat. Tech. 207, 310 (2012).

[6]. Y.C. Lin, S.C. Chen, J. Mater. Process. Tech. 138, 22 (2003).

[7]. M.D. Toit, J.V. Niekerk, Weld. World 54, R342 (2010).

[8]. A. de Aquino, Y. Prasad, R.A. Sanguinetti, Mat. Res. 17, 295 (2014).

[9]. P. K. Palani, N. Murugan. Mater. Manuf. Process. 21, 431 (2006).

[10]. B. Abbasi-Khazaei, A. Mollaahmadi, J. Mater. Eng. Perform. 26, 1626 (2017).

[11]. S. Al Saadi, Y. Yi, P. Cho, C. Jang, P. Beeley, Corros. Sci. 111, 720 (2016).

(c) 2019 by the authors; licensee SMCTSM, Mexico. This article is an open access article distributed under the terms and

conditions of the Creative Commons Attribution license (http://creativecommons.org/licenses/by/4.0/).
[12]. Mark C. Biesinger, B. P. Payne, A. P. Grosvenor, L. W.M. Lau, A. R. Gerson, R. St.C. Smart, Appl. Surf. Sci. 257, 2717 (2011).

[13]. S. Tardio, M. L. Abel, R. H. Carr, J. E. Castle, J. F. Watts, $J$. Vac. Sci. Technol. A 33, 05E122-1 (2015).

[14]. Y. Yang, J. D. Scantlebury, E. V. Koroleva, Metals. 5, 439 $\underline{(2015)}$.

[15]. S. Elbeik, A. C. C. Tseung, A. L. Mackay, Corros. Sci. 26, 669 (1986).

[16]. N. Imaz, M. Ostra, M. Vidal, J.A. Díez, M. Sarret, E. GarcíaLecina, Corros. Sci. 78, 251 (2014).

[17]. H-K. Song, H-Y. Hwang, K-H. Lee, L.H. Dao, Electrochim.

[18]. C. Liu, A. Leyland, Q. Bi, A. Matthews, Surf. Coat. Tech.141, 164 (2001).

[19]. C. Liu, Q. Bi, A. Leyland, A. Matthews, Corros. Sci. 45, 1243 (2003). Acta 45, 2241 (2000). 